\title{
UNA MIRADA A LA COMPETITIVIDAD DEL SISTEMA BANCARIO NACIONAL COSTARRICENSE A TRAVÉS DEL INDICADOR DE BOONE 2008-2013
}

\author{
Andrés Alberto Salas Alvarado ${ }^{1}$ \\ Jose Carlos Mora Guerrero ${ }^{2}$ \\ Oscar Emilio Agüero Rodríguez ${ }^{3}$
}

Recibido: 30/06/2015

Aprobado: 16/11/2015

\begin{abstract}
RESUMEN
En esta investigación se estima el grado de competitividad del sistema bancario costarricense para el periodo 2008-2013. Para esto se utiliza el indicador de Boone, una propuesta no estructural que surge de la nueva teoría de la organización industrial. El estudio se aborda a partir de dos estimaciones. Primero, el indicador de Boone individual para cada entidad bancaria y segundo, un indicador mensual para el sistema agregado. Para realizar las estimaciones se utiliza la metodología sugerida por Griffith, Boone y Harrison (2005). Se concluye que el mercado no es competitivo, que las entidades bancarias privadas son en promedio más competitivas que las estatales y que durante el periodo de crisis se presenció una disminución en el grado de competitividad del sistema bancario costarricense.
\end{abstract}

PALABRAS CLAVE: COMPETENCIA, ELASTICIDAD DE LA GANANCIA, PODER DE MERCADO, MEDIDA DE COMPETENCIA, MERCADOS IMPERFECTOS, ESTRUCTURA DE MERCADO.

\section{ABSTRACT}

In this study, the degree of competitiveness of the Costa Rican banking system is estimated for the 2008-2013 period. For this, the Boone indicator is used, a non-structural proposal that has emerged from the new theory of industrial organization. The study is based on two estimations: first, the single Boone indicator for each bank, and second, a monthly indicator for the aggregate system. To estimate the indicator, the methodology suggested by Griffith, Boone and Harrison (2005) is used. The main conclusions are that the market is not competitive, private banking institutions are on average more competitive than public ones and the degree of competitiveness of the banking system decreased during the crisis period.

KEYWORDS: COMPETITION, PROFIT ELASTICITY, MARKET POWER, COMPETITION MEASURE, IMPERFECT MARKETS, MARKET STRUCTURE.

1 Copal Amba. Código postal 40104, Heredia, Costa Rica; andres.salas.a@gmail.com

2 Mckinsey \& Company, Código postal 40104, Heredia, Costa Rica; mg.jose92@gmail.com

3 Universidad de Costa Rica, IICE, Código postal 11501-2060, San José, Costa Rica; osagrodriguez@gmail.com 


\section{INTRODUCCIÓN}

En esta investigación se estima el grado de competitividad del sistema bancario costarricense para el periodo 2008-2013, para esto se utiliza el indicador de Boone aplicando la metodología sugerida por Griffith, Boone y Harrison (2005). Esta metodología comprende una regresión lineal de la razón del resultado operacional neto a activo total, en función de los costos marginales. Las estimaciones se abordan desde dos perspectivas, para las entidades bancarias individuales se estima el indicador de Boone puntual para todo el periodo, esto permitió concluir cuales son las entidades más competitivas del sistema. Para el sistema agregado se estima un indicador de Boone mensual, lo que permitió observar la dinámica de la competitividad del sistema durante del periodo analizado, periodo que coincide con una crisis económica. Se concluye que el mercado no es competitivo, que las entidades bancarias privadas son en promedio más competitivas que las estatales y que durante el periodo de crisis se presenció una disminución en el grado de competitividad del sistema bancario costarricense.

Respecto a la literatura económica sobre competencia y estructuras de mercado, se puede mencionar que desde la organización industrial se han desarrollado dos corrientes para su estudio. La estructural y la no estructural, dentro de la primera se enmarcan los estudios de estructura-conducta-desempeño. Estos se caracterizan por otorgarle gran importancia a la concentración de los mercados, es decir que se basan en la premisa de que una alta concentración causa una conducta menos competitiva en las firmas y genera mayores ganancias para los participantes (Degryse, Kim, y Ongena, 2009).

Por su parte los estudios no estructurales evalúan la competencia y el uso del poder de mercado en ausencia de medidas estructurales como la concentración. Estos surgen como contra propuesta de los primeros modelos, especialmente por la deficiencia de las medidas de concentración para medir la competencia ${ }^{4}$ (Bikker y Haaf, 2002).

Para el sistema bancario y financiero costarricense, los principales estudios han sido elaborados a partir del enfoque no estructural. Por ejemplo Laverde y Madrigal (2005), utilizando el modelo de Bresnahan (1982), concluyen que para el periodo 1997-2005 el sistema bancario costarricense no es competitivo, por el contrario presenta cierto poder monopólico, especialmente cuando se trata de bancos estatales.

Por su parte Durán, Quirós y Rojas (2009), concluyen a través de los modelos de Panzar-Rosse (1987) y Shaffer (1993) que para el periodo 1995-2009 la estructura del sistema financiero costarricense es oligopólica y que a pesar de que la actividad de intermediación se puede asumir como homogénea, los participantes del mercado siguen estrategias para diferenciarse de los demás y así ejercer cierto poder sobre los precios. A diferencia del estudio de Laverde y Madrigal (2005), en esta investigación, además de las entidades bancarias, se incorporan todos los intermediarios que conforman el Sistema Financiero Nacional.

Empleando un modelo no estructural de maximización de beneficio bancario, Castro y Serrano (2013), concluyen que para el periodo 2000-2012 el sistema bancario costarricense no es competitivo, al contrario lo clasifican como mercado oligopólico al estilo Stackelberg, en el que las entidades toman sus decisiones de forma secuencial y no simultáneamente, siendo la banca privada seguidora de la estatal.

Por último desde el enfoque de economías de escala, ámbito y poder de mercado, Gutiérrez (2013), concluye que para el periodo 1997-2009, del mercado bancario, las entidades públicas presentan costos marginales menores que las privadas, lo cual les permite la colocación de crédito, inversiones y servicios en el mercado de una forma mucho más fácil competitiva. Se determina que el Banco Nacional de Costa Rica y el BAC San José son los bancos con mayores economías de escala. 


\section{METODOLOGÍA}

El indicador de Boone parte de la premisa de que entre más eficientes sean las entidades bancarias (menor costo marginal) más altas serán sus ganancias, consecuentemente más competitivo será el sistema bancario, esto como resultado de que la conducta agresiva en el mercado elimina las firmas ineficientes, incrementando así la concentración. El indicador expresa una relación negativa entre las ganancias y el costo marginal, entre más negativa esta relación, más competitivo será el mercado o las entidades analizadas. El modelo parte de una curva de demanda para las entidades bancarias i, que producen un producto qi con un costo marginal $\mathrm{cm}$, esta curva se expresa de la siguiente forma:

$$
p\left(q_{i}, q_{j \neq i}\right)=a-b q_{i}-d \sum_{j \neq i} q_{j}
$$

De acuerdo a Boone (2008), la maximización de las ganancias se da cuando las entidades definen un nivel óptimo de producto, asumiendo que $a>c m_{i}$ y $0<d \leq b$. Obteniendo la condición de primer orden para el equilibrio de Cournot-Nash, la ecuación se puede re escribir de la siguiente forma:

$$
a-2 b q_{i}-d \sum_{j \neq i} q_{i}-c m_{i}=0
$$

Cuando $\underline{\mathrm{N}}$ bancos obtienen niveles positivos de producto, se pueden resolver las $\underline{\mathrm{N}}$ condiciones de primer orden, obteniéndose la siguiente expresión:

$$
q_{i}\left(c m_{i}\right)=\frac{\left[\left(2 \frac{b}{d}-1\right) a-\left(2 \frac{b}{d}+N-1\right) c m_{i}+\sum_{j} c m_{j}\right]}{\left[(2 b+d(N-1))\left(2 \frac{b}{d}-1\right)\right]}
$$

Siguiendo a Boone (2008), en los mercados la competitividad se va a incrementar de dos formas. Primero, cuando los servicios de los bancos se vuelven sustitutos cercanos, esto es, un incremento en $d$, siendo d menor que b. Y segundo, cuando se reducen los costos de entrada. Por lo tanto una forma sencilla de expresar esta relación negativa entre ganancia y costo marginal puede definirse de la siguiente forma.

$$
\pi=\alpha+\beta(\mathrm{cm})
$$

Donde $\pi$ representa la ganancia, $\beta$ la elasticidad de la ganancia o indicador de Boone $y \mathrm{~cm}$ el costo marginal.

Para realizar la estimación empírica se adopta la propuesta de Griffith, Boone y Harrison (2005), quienes sugieren utilizar el costo variable medio $\frac{c v t}{Y}$ como proxy del costo marginal, esto como consecuencia de que el costo marginal es una variable no observable. Si bien el costo marginal se puede obtener a través de otros métodos como el método generalizado de momentos o mínimos cuadrados ordinarios mediante funciones translogaritmicas de costo, por tratarse de una primera aproximación al indicador de Boone estos métodos son obviados y se dejan como una oportunidad para futuras investigaciones. 


$$
\begin{aligned}
\pi & =\alpha+\beta\left(\frac{c v t}{Y}\right) \\
c m & \cong\left(\frac{c v t}{Y}\right) \\
\left(\frac{c v t}{Y}\right) & =\left(\frac{C^{*} Q}{P^{*} Q}\right)=\frac{C}{P} \cong c m
\end{aligned}
$$

En (7) se tiene que el costo variable total es igual al costo de cada unidad multiplicado por el número de unidades adquiridas, $y$ el ingreso es igual al precio de venta multiplicado por el número de unidades vendidas. Consecuentemente la variable $Q$ se puede cancelar.

Ahora, partiendo del índice de Lerner se obtiene la siguiente expresión:

$$
I L=\frac{P-c m}{P}=1-\frac{c m}{P}
$$

Despejando el costo marginal $\mathrm{Cm}$ la ecuación se re expresa de la siguiente forma:

$$
\frac{c m}{P}=1-I L
$$

La ecuación (8) muestra que las firmas más eficientes poseen un índice de Lerner más alto, por lo tanto se enfrentan a un coeficiente $\frac{\mathrm{cm}}{P}$ menor. Así se muestra la relación entre el costo marginal $y$ la variable proxy $\frac{C}{P}$.

La estimación del indicador se realiza mediante el método de mínimos cuadrados ordinarios a través del siguiente modelo de series de tiempo.

$$
\pi_{t}=\beta_{1}+\beta_{2} \operatorname{Ln}\left(c m_{t}\right)+\varepsilon_{t}
$$

Donde $\pi_{t}$ es la ganancia, $\mathrm{cm}_{t}$ el costo marginal $y \varepsilon_{t}$ un ruido blanco. Se debe considerar que algunas entidades bancarias presentan resultados operacionales negativos para varios periodos, por lo que la regresión lineal se estima de la forma Lin-Log para evitar inconvenientes con logaritmos negativos. Por lo tanto, el indicador de Boone o elasticidad de la ganancia se obtiene a través del cociente $\frac{\beta_{2}}{\gamma}$, siendo $\gamma$ el promedio de $\pi_{t}$. Las variables operacionales que se utilizan para realizar las estimaciones son las siguientes.

CUADRO 1

DEFINICIÓN DE VARIABLES

\begin{tabular}{ll}
\hline Variable & Construcción de la variable \\
\hline Ingreso & Ingreso por intermediación financiera \\
& $(+)$ Ingreso por comisiones y servicios \\
& Gasto de personal \\
Costo variable total & $(+)$ Gastos por comisiones y prestación de servicios \\
& $(+)$ Gastos por intermediación \\
Ganancia & Resultado Operacional neto \\
& $(\div)$ Activo Total \\
Costo variable Medio & Costo variable Total \\
\hline
\end{tabular}

Fuente: elaboración propia. 
Los datos utilizados para estimar los modelos se obtuvieron del sitio web de la Superintendencia General de Entidades financieras. Se utilizan los estados financieros en formato de análisis financiero ${ }^{5}$ y el periodo considerado para la estimación es del año 2008 al 2013 de forma mensual para un total de 72 observaciones. Las entidades bancarias consideradas se muestran en el siguiente cuadro. Es importante considerar que algunas entidades presentan problemas para cumplir los supuestos del modelo de regresión lineal por lo que se hace algunos ajustes en las muestras, el anexo 6 muestra cada una de las muestras seleccionadas.

\section{CUADRO 2}

\begin{tabular}{llll}
\hline Lista de entidades bancarias consideradas para el estudio & & \\
\hline Banco Nacional de Costa Rica & Scotiabank & Banco Lafise & Banco General \\
Banco de Costa Rica & Banco Davivienda & BAC San José & Banco Citibank \\
Banco Popular y de Desarrollo Comunal & Banco de soluciones & Banco BCT & Banco Improsa \\
Banco Crédito Agrícola de Cartago & Banco Promerica & Banco Cathay & \\
\hline
\end{tabular}

Fuente: Lista de entidades supervisadas por SUGEF.

Para analizar el comportamiento del indicador de Boone para el sistema bancario agregado durante el periodo 2008-2013, se estima una regresión progresiva con el software E-Views, esta regresión genera los valores del indicador mensualmente (ver anexo 3), este procedimiento logra modelar la dinámica de la competitividad del sistema bancario a través de la muestra definida. Posteriormente se estiman correlaciones cruzadas, con adelantos y rezagos, respecto a la tasa de crecimiento del crédito para determinar si existe una relación entre estas variables. Las correlaciones se calculan de la siguiente forma:

$$
\rho_{i}=\operatorname{corr}\left(\beta_{t}, C r_{t+i}\right), \text { para } i=-k,-k+1, \ldots, 0, \ldots, k-1, k
$$

Donde $\mathrm{Cr}$ es el Crecimiento del crédito y $\beta_{t}$ el indicador de Boone.

\section{RESULTADOS}

De las estimaciones realizadas se obtiene que las entidades bancarias con mayores participaciones de mercado ${ }^{6}$, poseen indicadores de Boone mayores al promedio del sistema (-2.57), es decir los indicadores son menos competitivos. Esta situación se observa en tres de las cuatro entidades bancarias estatales. El Banco Nacional, Banco Popular y Banco de Costa Rica, por lo tanto únicamente el Banco Crédito Agrícola de Cartago posee un grado de competitividad superior al del promedio del sistema.

5 El formato para análisis presenta algunas modificaciones contables en las partidas de los estados financieros, resume el saldo de los principales rubros de activos, pasivos y capital, de un sector, una entidad o varias entidades, a una fecha determinada, efectuando algunas agrupaciones de la información específicamente para efectos de análisis financiero.

6 La participación de mercado se define como el cociente de la cartera de crédito total de la entidad i dividida por la sumatoria de todas las carteras totales del sistema. 
CUADRO 3

PARTICIPACIÓN DE MERCADO PROMEDIO E INDICADOR DE BOONE SISTEMA BANCARIO COSTARRICENSE 2008-2013

\begin{tabular}{lrrr}
\hline Entidad Bancaria & $\begin{array}{c}\text { Banca Estatal } \\
\text { \% Mercado } \\
\text { Banca Estatal }\end{array}$ & & Indicador de Boone \\
\hline Banco Crédito Agrícola & & 2,74 & $-3,52$ \\
Banco Popular & 13,17 & $-2,54$ \\
Banco de Costa Rica & 19,05 & $-2,46$ \\
Banco Nacional & 26,24 & $-1,55$ \\
Media & & $-2,52$ \\
\hline & Banca Privada & & \\
\hline Banco de Soluciones & & 0,33 & $-7,77$ \\
Banco Cathay & & 0,48 & $-6,49$ \\
Banco Lafise & 1,58 & $-3,81$ \\
Banco Promerica & 2,41 & $-2,88$ \\
Banco Improsa & 1,78 & $-2,60$ \\
Citibank & & $-2,13$ \\
BAC San José & & 11,36 & $-1,94$ \\
Banco BCT & 1,26 & $-1,71$ \\
Davivienda & 4,30 & $-1,23$ \\
Banco General & & $-1,23$ \\
Scotiabank & 0,84 & 3,25 \\
Media & 9,79 & $-2,59$ \\
\hline
\end{tabular}

Fuente: elaboración propia.

Por su parte los resultados de la banca privada muestran que cinco de once entidades superan el promedio de competitividad del sistema. De estas cinco ninguna supera el 3\% de participación de mercado promedio para el periodo 2008-2013, siendo el Banco Promerica el de mayor participación de mercado con un $2.41 \%$. De estos resultados se puede afirmar que la banca estatal es en promedio menos competitiva que la banca privada.

Desde la perspectiva del sistema en su conjunto se observa que durante el periodo analizado el grado de competitividad ha sufrido cambios importantes, llegando a estar muy cerca de ser positivo a finales del año 2011. A partir de mayo del 2009 y hasta enero del 2012 el indicador incrementa $y$ desde febrero del 2012 el indicador tiende a un comportamiento similar al del inicio del 2008 (ver anexo 1).

Por otra parte, los resultados de las correlaciones cruzadas con la tasa de crecimiento del crédito muestran que la correlación más fuerte se da con un rezago de cuatro periodos. Si bien no se puede establecer causalidad, se observa una relación bastante sugestiva, que señala que el impacto de la reducción del crédito en la competitividad del sistema bancario es rezagado con un lapso entre tres y cinco periodos, como lo muestra el siguiente gráfico. 
GRÁFICO 1

CORRELACIONES CRUZADAS ENTRE LA TASA DE CRECIMIENTO DEL CRÉDITO Y EL INDICADOR DE BOONE

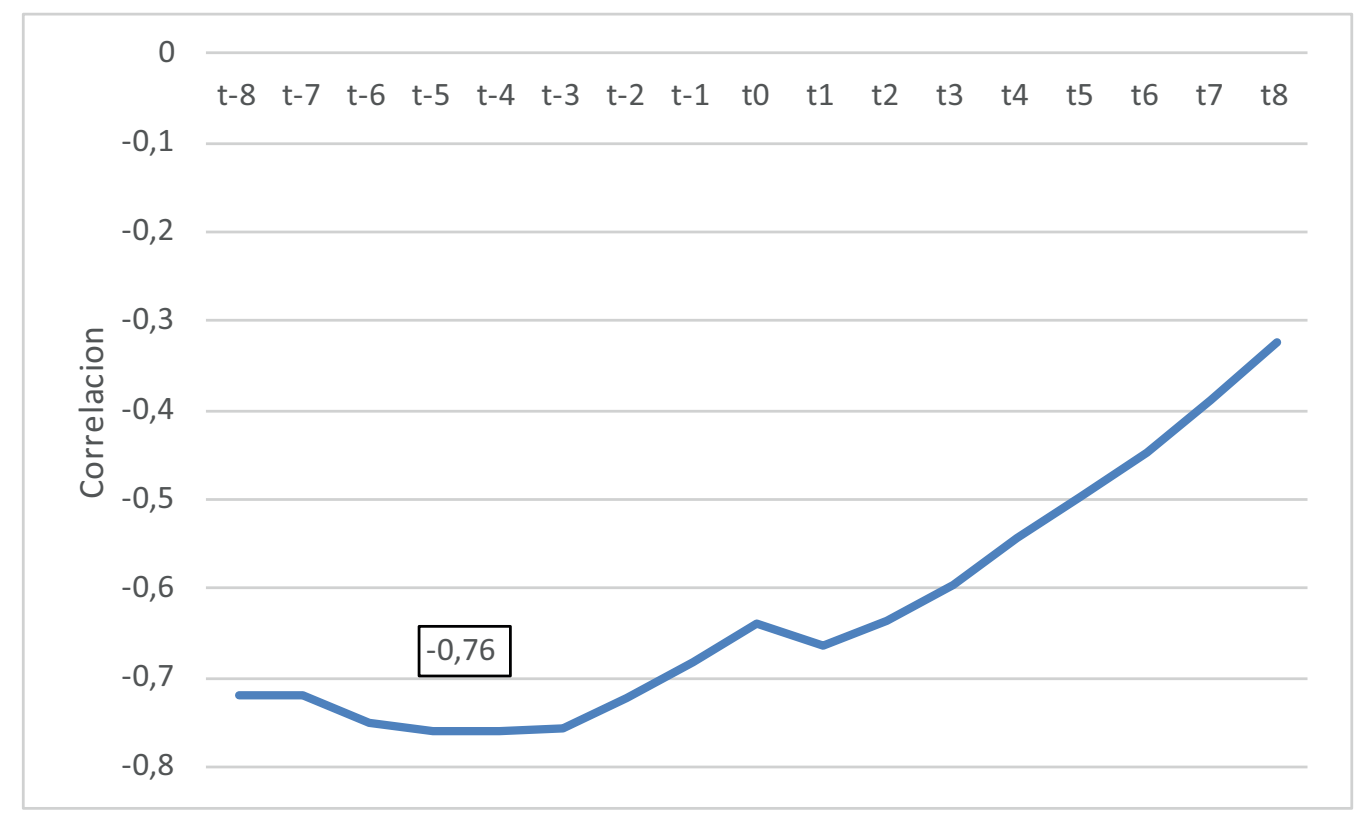

Fuente: elaboración propia.

La correlación negativa observada entre el crecimiento del crédito y el indicador de Boone puede surgir como resultado del deterioro de la economía, las entidades bancarias disminuyen sus colocaciones y por ende sus ganancias. El indicador de Boone se torna cada vez más positivo como consecuencia de que las entidades no transfieren ganancias de eficiencia a los clientes, ya que además de la disminución de las colocaciones, la transferencia de eficiencia en un periodo de crisis empeoraría la situación financiera de las entidades.

\section{CONCLUSIONES Y RECOMENDACIONES}

De los resultados de las estimaciones individuales se concluye que el mercado bancario costarricense no es competitivo, como ya indicaban estudios anteriores. Esto como consecuencia de que las firmas con mayor participación de mercado poseen indicadores de Boone poco competitivos.

Los resultados indican que la banca privada es en promedio más competitiva en el sentido de Boone que la banca estatal. Por lo general, los primeros presentaron indicadores menores, esto tiende a coincidir con los márgenes de intermediación financiera, los cuales son menores en la banca privada (ver anexo 2).

El hecho de que las entidades con mayores participaciones de mercado sean parte del grupo de las menos competitivas sugiere que emplean otro tipo de estrategias para captar mayor participación de mercado, por ejemplo la diferenciación del producto, ya sea de forma vertical u horizontal.

A través de las regresiones generadas mediante un loop en el software E-views fue posible obtener el indicador de Boone mensual del sistema bancario para el periodo 2008-2013. Esto permitió observar un deterioro en el nivel de competencia del sistema bancario agregado, deterioro que coincide con el periodo de la última crisis económica del país. Consecuentemente, para 
investigaciones futuras se hace necesario profundizar en el efecto de los shocks externos sobre la estructura del mercado bancario costarricense.

Desde la perspectiva de las políticas para fomentar la competencia en el sistema bancario, desde el enfoque de Boone, deben estar orientadas incrementar el grado de sensibilidad en el indicador ante cambios en el costo marginal de las entidades. Por ejemplo, el incremento de sustituibilidad entre productos, sumado a la minimización de información asimétrica que enfrentan los consumidores. De forma que los agentes puedan percibir los incrementos en los costos marginales. Esto a través de la divulgación de información periódica, atractiva para el consumidor promedio, que contenga aspectos como costos reales, calidad, condiciones.

Por otro lado, es importante fomentar una cultura bancaria más desarrollada en el costarricense, de forma que racionalice sus decisiones de consumo financiero, es decir que sea capaz de realizar un análisis que le permita determinar cuáles son las condiciones del servicio que más convienen para sus condiciones económicas. En esencia la línea de la política debe dirigirse a incrementar la racionalidad del consumidor financiero.

Finalmente, el estudio arroja resultados interesantes desde un enfoque diferente al utilizado en estudios anteriores, sin embargo, quedan aspectos que pueden ser mejorados e incorporados en futuras investigaciones. Desde de la teoría de la organización industrial, se hace necesario profundizar dos aspectos. Los factores que generan niveles altos de ganancia en bancos con indicadores de Boone poco competitivos, tales como la diferenciación de productos, la garantía estatal que carece la banca privada e incluso factores institucionales como el decreto de nacionalización bancaria. Y la relación empírica entre ciclos económicos y el grado de competencia en el sistema bancario costarricense.

\section{AGRADECIMIENTOS}

Agradecemos las valiosas sugerencias del señor Jorge Madrigal del Consejo Monetario Centroamericano, cualquier error u omisión es responsabilidad de los autores. Las opiniones expresadas en el documento son propias de los autores y no necesariamente representan la posición de las instituciones en las que laboran.

\section{REFERENCIAS}

Bikker, J., y Haaf, K. (2002). Measures of Competition and Concentration in the Banking Industry: a Review of Literature [Medidas de la Competencia y de la concentración en el sector bancario: una revisión de la literatura]. Economic and Financial Modelling , 1-46.

Boone, J. (2008). A New Way to Measure Competition. [Una nueva forma de medir la Competencia]. The Economic Journal, 118(531), 1245-1261. doi: 10.1111/j.1468-0297.2008.02168.x.

Castro, A., y Serrano, A. (2013). Margen de Intermediación Financiera y Poder de Mercado: el Caso de Costa Rica. San José: Banco Central de Costa Rica.

Degryse, H., Kim, M., y Ongena, S. (2009). Microeconometrics of Banking; Methods Applications and Results. [Microeconometría de la Banca; Métodos Aplicaciones y Resultados]. New York: Oxford University Press. doi: 10.1093/acprof:oso/9780195340471.001.0001.

Durán, R., Quirós, J., y Rojas, M. (2009). Análisis de la Competitividad del Sistema Financiero Costarricense. San José: Banco Central de Costa Rica.

Griffith , R., Boone, J., y Harrison R. (2005). Measuring Competition. [Medición de la competencia]. Advanced Institute of Management Research.

Gutiérrez, A. (2013). Competencia en la Industria Bancaria de Costa Rica: un Enfoque desde las Economías de Escala para el Periodo 1997-2009. Ciencias Económicas, 31(1), 139-152.

Laverde, B., y Madrigal, J. (2005). Identificación del Grado de Competencia en el Mercado Bancario Costarricense. San José: Banco Central de Costa Rica. 
ANEXOS

ANEXO 1

INDICADOR DE BOONE SISTEMA BANCARIO COSTARRICENSE 2008-2013

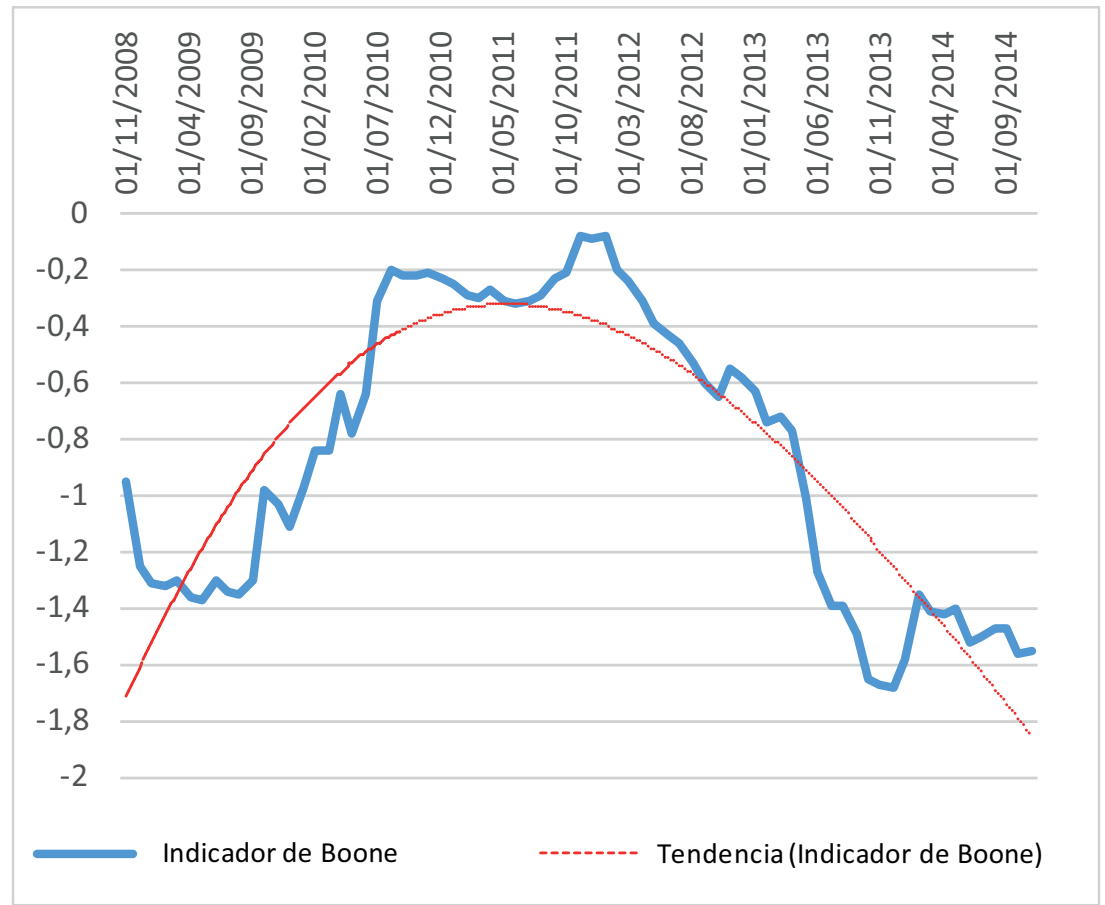

Fuente: elaboración propia. 
ANEXO 2

MARGEN DE INTERMEDIACIÓN IMPLÍCITO SISTEMA BANCARIO COSTARRICENSE 2008-2013

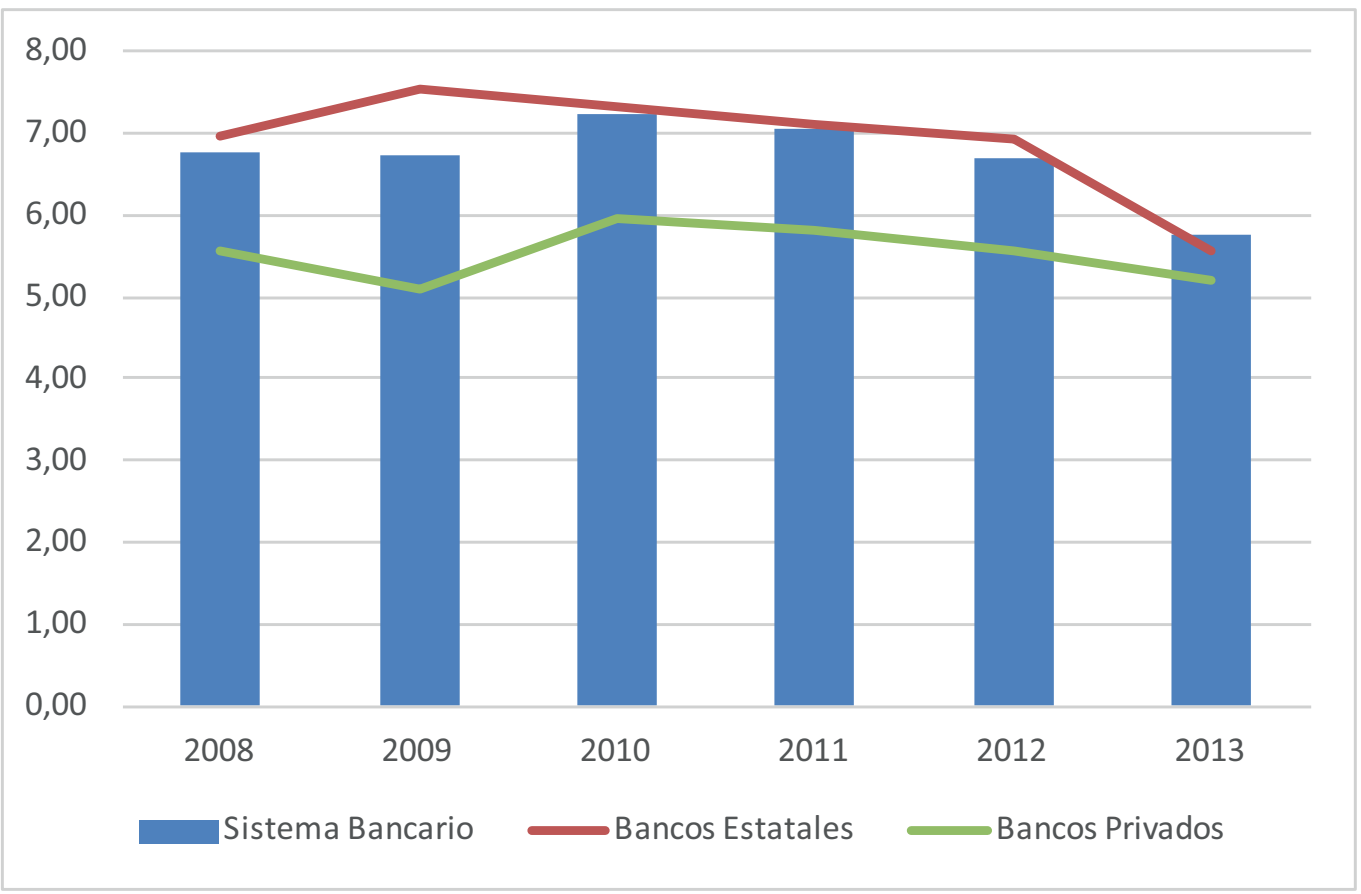

Fuente: elaboración propia con datos de SUGEF.

ANEXO 3

REGRESIÓN SISTEMA BANCARIO CON LOOP EN E-VIEWS

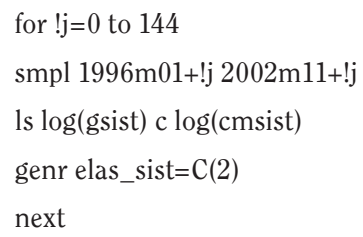

Fuente: elaboración propia. 
ANEXO 4

INDICADOR DE BOONE SISTEMA BANCARIO COSTARRICENSE Y TASA DE CRECIMIENTO DEL CRÉDITO 2008-2013

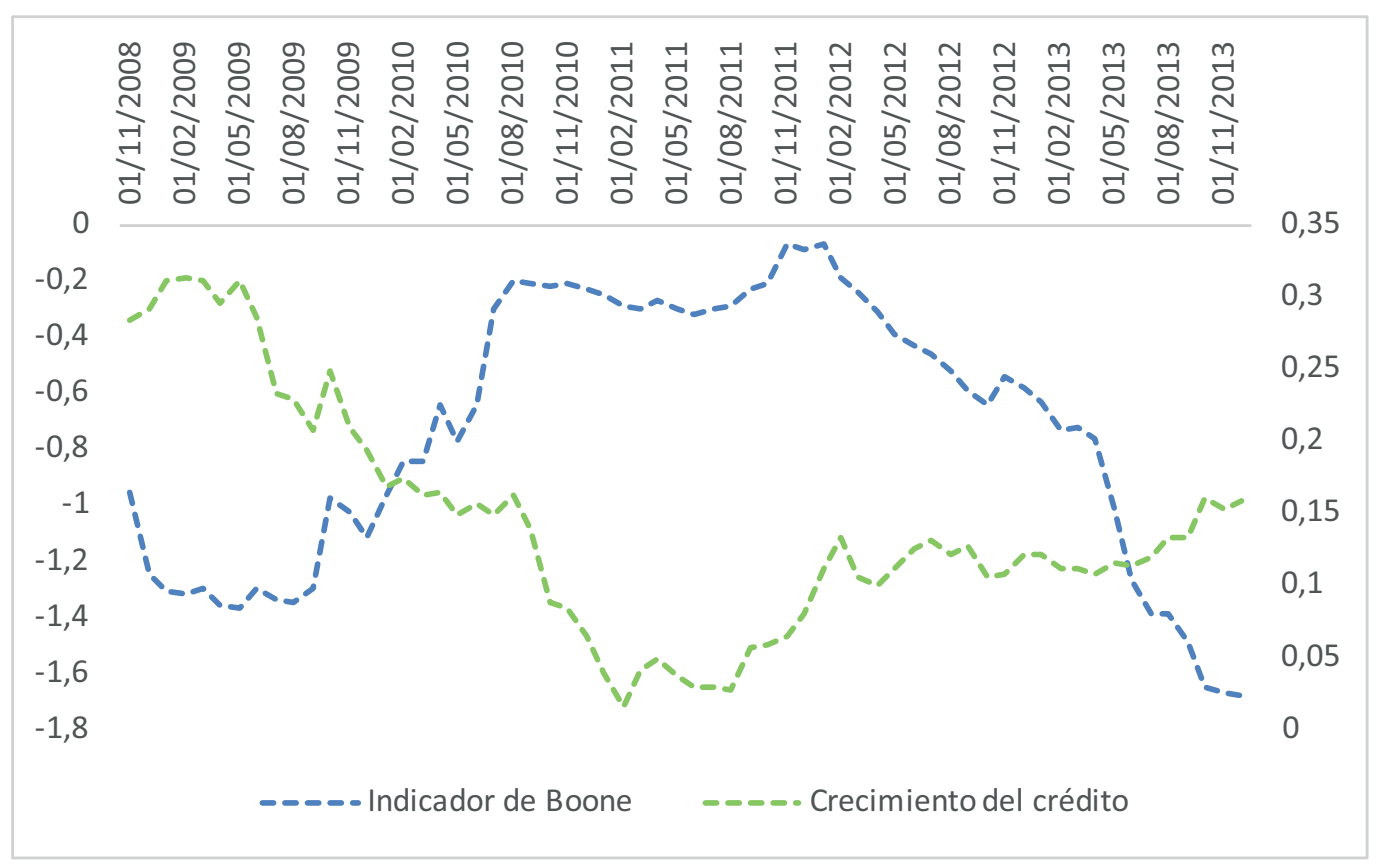

Fuente: elaboración propia con datos de SUGEF. 
ANEXO 5

RESULTADOS ESTIMACIÓN INDICADOR DE BOONE 2008 - 2013 SISTEMA BANCARIO COSTARRICENSE

\begin{tabular}{|c|c|c|c|c|c|c|c|c|c|c|}
\hline$\alpha$ & Banco & Muestra & $\begin{array}{c}\text { R2 } \\
\text { Ajustado }\end{array}$ & B0 & $\mathrm{P}$ & B1 & $\mathrm{P}$ & B-G & B-G-P & $\mathrm{J}-\mathrm{B}$ \\
\hline $1 \%$ & Banco de Soluciones & $2011 \mathrm{~m} 1-2014 \mathrm{~m} 12$ & 0,718 & $-0,001$ & 0,000 & $-0,008$ & 0,000 & 0,506 & 0,938 & 0,000 \\
\hline $1 \%$ & Banco Cathay & $2010 \mathrm{~m} 10-2014 \mathrm{~m} 11$ & 0,471 & 0,000 & 0,037 & $-0,004$ & 0,000 & 0,081 & 0,537 & 0,707 \\
\hline $1 \%$ & Banco Lafise & $2009 \mathrm{~m} 1-2014 \mathrm{~m} 03$ & 0,580 & $-0,001$ & 0,259 & $-0,003$ & 0,001 & 0,902 & 0,281 & 0,010 \\
\hline $1 \%$ & $\begin{array}{l}\text { Banco Crédito } \\
\text { Agrícola }\end{array}$ & $2008 \mathrm{~m} 02-2013 \mathrm{~m} 12$ & 0,419 & 0,000 & 0,234 & $-0,004$ & 0,000 & 0,533 & 0,429 & 0,126 \\
\hline $1 \%$ & Banco Promerica & $2008 \mathrm{~m} 1-2013 \mathrm{~m} 12$ & 0,403 & $-0,002$ & 0,248 & $-0,007$ & 0,003 & 0,556 & 0,921 & 0,000 \\
\hline $10 \%$ & Banco Improsa & $2008 \mathrm{~m} 1-2013 \mathrm{~m} 12$ & 0,360 & 0,000 & 0,787 & $-0,003$ & 0,066 & 0,155 & 0,032 & 0,039 \\
\hline $1 \%$ & Banco Popular & $2008 \mathrm{~m} 1-2013 \mathrm{~m} 12$ & 0,723 & $-0,001$ & 0,610 & $-0,007$ & 0,000 & 0,417 & 0,571 & 0,321 \\
\hline $1 \%$ & Banco de Costa Rica & $2008 \mathrm{~m} 1-2013 \mathrm{~m} 12$ & 0,536 & 0,000 & 0,867 & $-0,004$ & 0,000 & 0,187 & 0,888 & 0,000 \\
\hline $1 \%$ & Banco Citibank & $2008 \mathrm{~m} 1-2013 \mathrm{~m} 12$ & 0,516 & $-0,001$ & 0,258 & $-0,004$ & 0,000 & 0,504 & 0,118 & 0,007 \\
\hline $1 \%$ & BAC San José & $2009 \mathrm{~m} 10-2014 \mathrm{~m} 11$ & 0,457 & $-0,002$ & 0,245 & $-0,006$ & 0,006 & 0,206 & 0,561 & 0,003 \\
\hline $10 \%$ & Banco BCT & $2008 \mathrm{~m} 1-2013 \mathrm{~m} 12$ & 0,047 & 0,000 & 0,829 & $-0,002$ & 0,077 & 0,430 & 0,901 & 0,001 \\
\hline $10 \%$ & Banco Nacional & $2008 \mathrm{~m} 1-2013 \mathrm{~m} 12$ & 0,460 & 0,000 & 0,602 & $-0,002$ & 0,000 & 0,072 & 0,392 & 0,541 \\
\hline $15 \%$ & Banco Davivienda & $2008 \mathrm{~m} 1-2013 \mathrm{~m} 12$ & 0,355 & 0,000 & 0,587 & $-0,002$ & 0,124 & 0,165 & 0,860 & 0,002 \\
\hline $1 \%$ & Banco General & $2011 \mathrm{~m} 1-2014 \mathrm{~m} 11$ & 0,397 & 0,001 & 0,001 & $-0,001$ & 0,000 & 0,723 & 0,789 & 0,590 \\
\hline $5 \%$ & Scotiabank & $2007 \mathrm{~m} 11-2013 \mathrm{~m} 12$ & 0,068 & 0,002 & 0,001 & 0,003 & 0,040 & 0,250 & 0,162 & 0,006 \\
\hline
\end{tabular}

Fuente: elaboración propia. 